\title{
11 Making things matter
}

\author{
Daniel Miller and Laura Haapio-Kirk
}

\section{From 'meaning' to 'mattering'}

One of the most concerning trends in global politics today is the way in which fear of the 'other' is operationalised for political gain. From the antiimmigration rhetoric of the pro-Brexit campaign, to garnering support for Trump's wall, the weapon of choice is often cultural intolerance. In many countries around the world stagnating economies give rise to increasing socio-economic and educational inequalities, which only fuels tensions. These conditions represent a historical moment that challenges the optimism of the Enlightenment (Pinker 2018), one of the main instruments of which has always been education itself. It is clear that in such circumstances a commitment to an engaged public anthropology matters. Furthermore, it is now possible for an anthropological education, and the humanistic principles it instils, to reach more people in this digitally connected age than ever before. It seems therefore a simple matter of responsibility that we directly engage with the question of how work in material culture and digital anthropology can actually matter beyond the academy. If anthropology is reduced to a discipline that justifies itself only as a 'critical anthropology,' but which merely critiques what is going on in the world for the benefit of other anthropologists, it is of little surprise if anthropology is largely ignored in the public sphere. Yet the current institutional forms of evaluation that (especially early career) anthropologists are subject to, are forces that tend to valorise this self-serving critique.

This chapter argues that we can address these issues directly through a focus upon what matters and to whom, and it demonstrates through case studies ways we can make our work matter. We document several stages in the trajectory of material culture studies and digital anthropology towards this goal of making things matter. A sub-theme will be the tension between things that matter to academia and things that matter to the populations we study. We start with a shift in anthropology from an emphasis on what things mean, to how they matter to our research participants. The next part examines how our research itself might matter to those same populations. The final part examines the pressures placed upon UK universities by the UK government's 'impact' agenda, and it proposes an alternative evaluation framework that 
also goes beyond education to include welfare as understood by the people we study. Finally, we consider how an alternative to impact evaluation can be an integral part of research design through the use of ethnography.

Modern material culture studies developed during the late 1970s and 1980s, inspired by the appropriation of new semiotic and structuralist approaches for the study of objects. Levi-Strauss's (1982) The Way of the Masks employed masks as analogous to myth in revealing structural transformations across societies. Books by Douglas and Isherwood (1978) and Sahlins (1976) addressed objects as categories, while Appadurai (1986a) examined the social life of objects, and Bourdieu (1977) addressed the materiality of habitus. Both structuralism and structural Marxism, however, emphasised the significance of objects for academics, while tending to ignore their significance for ordinary people. There was little place here for intentionality or human agency.

At UCL Anthropology during this time, material culture studies focused on modes of production (Friedman and Rowlands (eds) 1977); for the context see Godelier 1972; Hindess and Hirst 1975). Miller's book, Material Culture and Mass Consumption (1987), sought to re-think consumption from being conceived as the end point of production and distribution to being an active process of appropriation. In the introduction to the edited volume, Material Cultures (1998), titled, 'Why Some Things Matter,' Miller proposed a shift from an emphasis in semiotic and structuralist writing upon what things mean, to why things matter. The value of this term 'matter' was that it referred both to materiality and also to understanding why objects were important to people. Chapters in this volume examined material often considered trivial, such as paper in the office (Pellegram 1998) or shopping catalogues (Clarke 1998), and showed how they could be crucial in determining action and influencing people. The intention of the volume, and indeed of the people involved in material culture studies at UCL at the time, was to forge a distinct area of study focussed on materials themselves, rather than reducing objects to their linguistic significance or a reflection of social relations.

In recent years, the additional challenge has been how to use the methods and ideas developed in the study of tangible materials for understanding often intangible digital culture. The MSc in Digital Anthropology was established at UCL Anthropology under the umbrella of material culture studies to do precisely that. Not only can we draw on methods - including ethnography, cultural comparison, object analysis, and attention to materiality - but a material culture approach to the digital also means that we can find footing in an established body of theory. For example, advances in the study of offline visual material can be applied to understanding visual content posted online. In addition, we consider the materiality of technology such as computers or smartphones and examine their position in the context of people's offline lives. Materiality is important when thinking about the novel ways in which humans and machines are becoming increasingly intimately connected, whether through bodily augmentation (Parkhurst 2012) or, conversely, in the ways that data is constructed through material practices (Walford 2017). In 
rapidly ageing societies such as Japan, where a decreasing workforce and an increasing demand for care has driven the development of care technologies, a focus on materiality becomes especially important for understanding the affective capacities of technologies such as Pepper the robot, designed to elicit a sympathetic response (White 2018). Yet it is not only in the design of robots that humanoid characters are employed to communicate care. Haapio-Kirk's fieldwork in Kyoto and rural Kōchi (2018-2019) shows how LINE, Japan's most popular messaging app, which combines text with cute character images ('stickers'), has become an important medium for practicing care at a distance. Daily contact through visual messaging allows for affective contact while reducing the burden of care.

\section{From 'us' to 'them'}

Two decades after its publication, the limitations of the arguments in Material Cultures about making things matter have become clear. A shift from understanding what things mean to why they matter to the people we study was insufficient. The next stage would be to move beyond why things matter, to why our research itself matters beyond the remit of academic anthropology. For our research to matter in this broad sense, it needs to first be accessible to the general public. This implies a commitment to an intelligible style of writing and also a shift in emphasis in what anthropologists choose to write about. Much contemporary anthropological writing has re-orientated itself to a concern with relatively esoteric academic interests. Anthropology has passed through a sequence of theoretical interests, from post-modernism, post-structuralism, actor-network theory, and now to ontology and the Anthropocene, each in turn providing a carapace of obfuscating terminology that can be exploited in the manner described by Bourdieu (1984b), as each generation of anthropological students look for professional employment by establishing their reputation for theoretical agility and intellectual prowess.

A fixation with theory as an end in itself, rather than as a route to understanding the substantive, returns us to a situation where anthropologists develop their self-interest at the expense of those responsibilities outlined at the start of this chapter. Furthermore, most anthropological teaching is directed to small audiences of university students, who themselves tend to over-represent metropolitan elites. The situation suggests an urgent need for counter mechanisms developed for the express purpose of turning academic insights into education for a much wider public. Only then can we say that our work matters to people other than ourselves. This task is made all the more important by continued threats to the funding of disciplines that the general public may feel do not matter very much and therefore perhaps should not be funded. Anthropology is particularly precarious in the UK as it has not been taught at school level after the abolition of the Anthropology A-level in 2018. Making our work interesting and accessible beyond academia is actually vital to keeping academic anthropology in existence. 
Two case-studies will be used here to illustrate the kind of processes material culture studies might adopt in order to facilitate a return of our research back to the original task of education and helping people to gain a more empathetic appreciation and understanding of other people around the world, which is perhaps the fundamental legitimation for anthropological practice. The first case-study is the Why We Post project, which was led by Miller and based at the Department of Anthropology UCL, concluding in 2017. The ambition to connect with a wider public is made much easier if we start with a topic of current concern and interest to that public. The topic of this research was the uses and consequences of social media - a topic that has relevance for a broad spectrum of audiences: parents worried about their children going online (Livingstone and Olafsson 2018), governments worried about security issues (Morozov 2012), or migrants trying to stay in touch with relatives (Dekker, Engbersen, Klaver, Vonk 2018). If we genuinely believe that the depth and commitment of ethnography leads to a more profound understanding of such transformations in the way that we communicate, then we need to convey these findings as quickly as possible to as many people as possible.

The Why We Post project comprised a team of nine anthropologists who simultaneously engaged in 15-month ethnographies in field sites that ranged from Brazil and Chile to India and China. Haapio-Kirk was employed in the final two years to develop the project's spectrum of public dissemination. From its inception, accessible and effective dissemination was one goal of the project, learning directly from the populations studied. For example, the project discovered that whether people were learning the skills of hairdressing in lowincome Brazilian settlements (Spyer 2017), or learning about car mechanics or celebrity gossip in China and India (McDonald 2016; Venkatraman 2017; Wang 2016) short videos were crucial, especially for people with low levels of literacy. In an associated project, Sheba Mohammid studied the impact of these short videos on informal learning in Trinidad. Why We Post therefore made the commitment to produce ten short videos from each field site. ${ }^{1}$

The primary product of ethnography is the monograph; no other format speaks to our ethnographic method of holistic contextualisation (Miller et al. 2016: 28-29). Each field site resulted in a monograph with identical chapter headings to facilitate comparison. Five strategies were designed to facilitate wide dissemination. First, in emulation of popular historical genres, these monographs focus on conveying our ethnographic experience and findings with an emphasis upon storytelling. Academic discussion is largely relegated to footnotes. Second, the monographs were written in jargon-free accessible language. Third, we made direct use of innovations in Open Access publishing. Academic work is already paid for through grants derived from taxation. There are no grounds for authors to be paid twice through royalties. All our volumes were therefore downloadable for free from UCL Press. They were also available in an enhanced online format, with hyperlinks to videos and other content. Fourth, it was important that the audience was not limited to English speakers, but that the books should be available in all the regions 
where these studies had taken place. So, apart from Turkish, where there are currently certain political constraints, all the volumes are being translated in the local fieldsite languages, as has the comparative volume, How the World Changed Social Media (Miller et al. 2016). The final strategy was to embed the monograph in a holistic schema of research dissemination that also included our active social media, our films, a website ${ }^{2}$ and our online course (MOOC).

The combination of these five strategies has proved highly effective. At the time of writing this chapter (July 2020) downloads for this series have passed one million. In 2018 books from the Why We Post series represented the first, second and fourth most popular open access book downloads, across all disciplines, on JSTOR, the primary online scholarly library containing 12 million sources from 75 disciplines ${ }^{3}$. Finally, and of particular concern to anthropology, the downloads are global, including many countries where sales of a printed ethnographic monograph were likely to have been very limited. For example, the first year's figures included 14,800 for the Philippines, and 5,068 for Ethiopia. The traditional ethnographic monograph usually sold in hundreds rather than thousands and it would be unusual to obtain any sales at all in many developing countries.

As part of this holistic research dissemination strategy we also created a free online course that could reach beyond the few dozen students we would normally address in a lecture theatre. Why We Post became the first MOOC created at University College London for the FutureLearn platform, which is part of The Open University. The MOOC movement represents one way in which technology has been harnessed for opening up education. MOOCS have received criticism for typically relying on videos and quizzes, and for not providing learners with a sense of community or enabling them to coconstruct their knowledge (McAuley et al. 2010), but FutureLearn works more like a social media platform with considerable interactivity between students. A Russian translation of the course has been used for teaching over six thousand students within Russia ${ }^{4}$ and a further translation into Thai has just been completed. At this point, more than twenty-two thousand students have been active participants on the Why We Post MOOC. This figure demonstrates the potential for MOOCs as a form of research dissemination that can bring anthropology to audiences who might not otherwise have considered the subject. Yet without institutional support, early career anthropologists face acute pressures to conform to job market demands which favour traditional lecturing over online teaching (Haapio-Kirk 2017). Finally, we also have a project led by Tom McDonald and Laura Poutney to incorporate the project findings within the school curriculum, initially in Hong Kong and within the UK as part of the A-level in Sociology by OCR, ${ }^{5}$ where it fits well within an already existing unit called 'Globalisation and the Digital World.' For this purpose, we held workshops with teachers and provided template classroom worksheets. ${ }^{6}$

Our second case study is intended to represent the other end of the spectrum from this largely digital global dissemination, examining instead an immersive and experiential example of research dissemination. In 2010 Inge 
Daniels published a book called The Japanese House based on many years' ethnography in Japan. Subsequently, in 2011, she was invited by the Geffrye Museum in London to create an exhibition, 'At Home in Japan.' She then returned to ethnography in order to assess the impact of her exhibition. The ethnography included observation of visitors as they interacted with the exhibition and each other, and post-visit interviews to assess their appraisal of the experience, conducted by Daniels and two research assistants, including Haapio-Kirk. The ethnography continued even after the exhibition ended, tracing the fate of the displayed objects, which were subsequently distributed through a raffle. This ethnography forms the foundation for another book, What are Exhibitions For (Daniels 2019). We will discuss how the impact of the exhibition was assessed in the next section.

The exhibition itself was intended to embody the principles that have been discussed here, in that the emphasis was on the possibilities of active consumption by the visitor rather than the production of the exhibition. Instead of treating material culture as art objects to be preserved behind glass cases, the exhibition was constructed as an ordinary home interior with the objects in drawers or on display as they would be in a typical Japanese apartment. The visitor could take them out, play with them, and if they removed them, these were cheap objects that could easily be replaced. Visitors were also asked to remove their shoes before stepping on tatami mats and behave in ways that would be expected of visitors to actual homes in Japan. The emphasis was on accessibility and intelligibility, based around stories of individual visitors. The educative possibilities included the provision of different depths of experience, since those wanting to have a deeper understanding could then read Daniel's ethnography. The style lends itself to popular education and also allows visitors more scope for exercising their own imaginations, which Daniels argues was one of the main consequences of this approach to exhibiting (Ibid. 201-203).

In this exercise, each element of exhibiting was reconsidered in terms of the potential for a more immersive experience. Instead of using photographs as framed artworks, in this exhibition they were full-sized and placed in a manner that extended the physical reconstruction of the house, or paired with displayed objects to help convey their original context (Ibid. 63-88). In turn, Daniels recognises that visitor photography has become an ever more important way that people interact with an exhibition, and her study examined where and why they take these photos and what happens to them after they are taken (Ibid. 171-193). So here the tradition of material culture studies has been employed directly to convey research findings through immersing the public in the material practices of other peoples of the world.

\section{Beyond impact}

All academics in the UK are today having to reimagine their work and its consequences in relation to a new agenda set by government. This is because a 
significant proportion of the funding of university departments now depends upon a demonstration that they have satisfied criteria that come under the label of 'impact.' What constitutes impact is decided by a series of rules and regulations. For example, the success of Why We Post in research dissemination cannot serve as direct impact, since the government's primary criteria is not educational, but evidence that other, non-academic bodies, have changed the way they behave as a result of this research. An example of impact would be that a commercial venture has put this knowledge into practice, such as marketing a product based on an anthropologically orientated semiotic analysis of that field of material culture. Many other countries have developed their own equivalent to the UK's impact agenda so that such concerns are becoming more important to academics world-wide.

The trajectory of this chapter has led to a point that is analogous, but also different, from the government's impact strategy. Having shown how we can study why things matter to people, and then how we can bring our findings back to people as education, we also face the issue of how we can make our work matter to people by contributing directly to the welfare of those populations. The problem is that currently we are doing so largely in response to the government's impact agenda. Instead, we need to formulate our own criteria for how things might matter for the wider world. We want to suggest two major departures from the government's impact agenda. The first is that anthropology should prioritise the welfare of the populations we study, and the second is that anthropology should contribute to ensuring that the populations themselves have a role in determining what we mean by 'welfare.' The example just given of helping a company improve its marketing would not satisfy either of those criteria.

What does it mean to suggest that populations should be included in this initial evaluation of what we mean by welfare? This proposal is derived from long-standing interventions by the economist Amartya Sen and philosopher Martha Nussbaum. Briefly, in opposition to the top-down determination of welfare embodied in the way economists or the United Nations ranked national welfare, Sen developed what became known as the capability approach: 'Our freedom to promote objectives we value' (Sen 1992: xi). But, as a paper on contraceptive choices by Mexican women (Beutelspacherm et al. 2003), published in Nussbaum's Feminist Economics (2003), showed, given the pressures people live under, it is very hard to understand what people would choose if given that capacity. Just as Sen showed that choice cannot be reduced to economic demand, anthropologists would argue that welfare values may not be reduced to choice - especially when we are talking about new digital technologies we have barely had time to assess. One legacy of anthropological work in structuralism and structural-Marxism is that we recognise that people make choices influenced by forces that they themselves may be quite unaware of. So merely asking people what they regard as their welfare would be insufficient. We would argue that it is actually ethnography, with its method of holistic contextualisation, that is best placed to assess the 
welfare goals of a given population. Furthermore, ethnography is rare in that it can accommodate contradictory ideals and practices, which is often the reality of welfare itself.

Soon after the completion of the Why We Post project, in 2017 Miller launched a new five-year project called ASSA - The Anthropology of Smartphones and Smart Ageing, ${ }^{7}$ also funded by the European Research Council. At first glance the idea of welfare intervention sounds like the traditional scope of applied anthropology, which often meant working with medical or other organisations to help fulfil welfare aims with respect to a given population. But this grant proposal was unusual. While it made a commitment to the general domain of smartphones and health, it did not specify what aspect of health or smartphone usage we would hope to develop interventions around. The principled reasoning behind this omission was that if before conducting any ethnography we had already decided how we would intervene in developing a population's welfare, we would have been imposing our own criteria and concerns upon that population. If, however, we want to follow the strictures of Sen and Nussbaum, then we need first to ensure that the population is a partner in establishing the appropriate welfare aims. For these reasons, discussion of possible interventions began only after we had completed ten months of ethnography. This allowed us to make many observations - for example, teaching smartphone usage to older people in order to better understand how they struggle with the new technologies. Smart ageing for us is understood as 'smart from below', the often ingenious appropriation of technology in everyday practice (Pype 2018), observable only through ethnography. The model came from Miller's previous study of the use of new media by hospice patients in the UK, which led to a series of suggestions as to how hospices night employ new media, all of which followed, rather than led, the research (Miller 2017).

We began writing this chapter precisely one year after the start of our ethnographies for the ASSA project: Miller in Ireland and Haapio-Kirk in Japan. This period has allowed for a natural evolution from the top-down perspective of writing a grant proposal to the bottom-up perspective based on the team's respective ethnographies. The original grant proposal mainly discussed potential engagement in terms of a huge industry that is churning out smartphone health apps. These generally follow from the interests and ideals of professional IT developers and medics, as well as commercial forces that recognise this is potentially a hugely profitable field. All three represent the top-down initiative normally associated with the word 'smart.' The topdown agenda also applies to academic work in this area, including that of anthropology. The dominant writing and interest in this field of smartphone health has so far been aimed at a particular set of practices that are associated with terms such as 'self-tracking' and 'data visualisation' (Dow Schull and Ruckenstein 2017). There are good reasons why academics find this a fascinating development. But in our ethnographies we see very little evidence that these constitute a significant aspect of mHealth (mobile health apps) in 
terms of actual consequences on populations. Some people use fitbits and step counters, but they are not widely used in most of our field sites, apart from Japan. One problem with current academic work is that it has tended to seek out and study the most extreme examples, such as people who belong to the 'Quantified Self' movement (e.g. Ajana Ed 2018; Lupton 2016). The problem is that such work takes the topics of most interest to academics and finds the requisite population.

By contrast, the ASSA project started with a deliberate attempt to forgo any academic agenda at all and, instead, allow the experience of ethnography to create a collaboration between the academic and those being studied to try to determine interventions based on aligning ourselves with what matters most to the people we work with as it emerges from that ethnography. As a result, over the course of the year the focus has largely moved away from dedicated smartphone health apps of interest to medical, technological and academic top-down agendas to focus instead on the usage of ubiquitous platforms such as Google and WhatsApp. Marilia Duque, working in Brazil, has developed a manual for health workers on the potential of WhatsApp for health, based on the observations she has made of both medical practitioners and her informants. We are also exploring the effects of Googling for health information. Charlotte Hawkins is working with an initiative led by Ugandan doctors rather than foreign NGOs in the assessment of possible mental health interventions in Kampala. Laura Haapio-Kirk is using her ethnography to unite patients, doctors, and state health officials in an effort to improve the experience of rural health care in Japan via the mobile phone. This sequence also ensures that the original ethnography retains its integrity as a project of enquiry, only after which we try to develop a strategy to make sure that the research matters.

ASSA's welfare goals are shared with many others, being akin to what Smith (2013) terms an 'interventionist design anthropology.' For example, the recent work of Jerome Lewis (2014), also based at UCL Anthropology, has extended the remit of digital anthropology at UCL in a similar direction. His team have worked with indigenous forest dwellers in West and Central Africa to develop a mobile application that enables them to report poaching and illegal logging, based on GPS and mobile photography. He classes his work under the umbrella of 'extreme citizen science,' which calls for as much involvement of the populations we study as possible in determining and securing their welfare. Research is conceived as a constant collaboration with participants in deciding what data should be collected, how it should be collected, and in determining its consequences. The chapter in this volume by Geismer approaches the issue of how to make Digital Anthropology matter through an engagement with the local community. There is, however, one final stage in this discussion of making things matter, in the context of the 'impact' agenda. To have our interventions accepted and valorised means we also have to consider how they will be evaluated. If we are unhappy with an increasing reliance on quantitative measures of evaluation, and we really 
want to demonstrate why anthropology matters, we should integrate methods of anthropology into impact assessment. The ASSA project is approaching potential mHealth interventions after a year's fieldwork, but it also includes provision for continuing to collaborate with the populations we work amongst for a further year after fieldwork, including an ethnographic assessment of those same interventions.

Fassin's edited collection (2017) presents the opportunities, challenges and limitations when ethnography 'goes public.' Yet, it remains the case that anthropologists have largely been absent from public debate, perhaps with the exception of Norway (Eriksen 2006, 2008). Despite anthropology's combination of deep and grounded observation with global comparative analysis, other disciplinary perspectives, notably from psychology and economics, often dominate public discourse (Ingold 2016). This may be a result of anthropology's withdrawal from public engagement during the twentieth century and its increasing institutionalisation (Eriksen 2016) . Encouragingly, today there is evidence of a growing movement by committed groups of anthropologists all over the world towards this endeavour, such as EASA's Applied Anthropology Network, which organises the annual conference, Why the World Needs Anthropologists. The authors in Fassin's collection demonstrate how ethnography has given them nuanced insight into often turbulent and precarious situations, where their intervention can have the potential to amplify under-represented voices. Yet there is an extra step that could demonstrate even further the value of anthropology: in the evaluation of public engagement itself. In the manner of Daniel's ethnography of her exhibition, the wider incorporation of ethnography of dissemination into our research design would allow us to learn directly from the public themselves how our efforts toward engagement are received, and ultimately how to do this better. So, in contrast to the government's Impact Agenda, anthropologists should consider the role of ethnography, both in determining what matters to a population, but also in the evaluation of our contribution to their welfare. Daniels's 2019 book demonstrates how an ethnographic evaluation of anthropological dissemination allows us to better understand how we might further innovate anthropological education by paying attention to this recursive nature of what matters.

\section{Conclusion}

This aim of this chapter was to demonstrate how a trajectory towards making things matter is not simply aspirational but is actually something we can hope to realise and that could drive material culture studies and digital anthropology in the future. The trajectory began in the 1970s, when after a long period in which material culture had been seen as peripheral to anthropology it started to emerge as of interest within structuralist and structural-Marxist perspectives. These perspectives established why objects might matter to academics but ignored the perspectives of the populations 
we study. Gradually, material culture studies at UCL developed an agenda that re-focused on why things mattered also to people other than academics. The next stage in this trajectory was to pay equal attention to the problem of how to make our research matter, which meant formulating strategies that could bring our research findings to the widest possible global audience and convince them that our work is significant and worth funding. For example, by making our work freely available, composed in accessible language and in an array of formats to suit different potential audiences. We have then moved on to consider how we can create an alternative to agendas such as the UK Impact policy. The ASSA interventions are intended to directly benefit the welfare of populations and also include that population's own understanding of what welfare means. Finally, we advocate for the use of ethnography also in the assessment of these contributions. This trajectory is not one of displacement; each of these goals is additional to the others, creating a more comprehensive model for the task of making things matter.

A sub-theme throughout this chapter has been the tension between whether things matter to academics or whether they matter to the population studied by academics. The problem we face was most fully theorised by the philosopher Hegel (2008) in his Philosophy of Right. All human institutions tend to migrate from the original legitimation for those institutions, usually in projects of reason, into something that is more self-serving. Law is supposed to adjudicate justice but can become a way lawyers amass a fortune. Shifting away from what matters to academics, or what is in their self-interest, does not mean reducing our aims to 'giving voice' to a population. Those earlier approaches from structuralism and structural-Marxism made a strong case for academics to excavate forces of power that populations may be entirely unaware of. Material culture ethnographies have never been mainly about asking people's opinions: they have always focused more on long-term observation of what people actually do, their practices, and extrapolating from our observations values that may not emerge in language. Our point is that the problem posed by Hegel in the Philosophy of Right will always be present. However well-intentioned, academic work will always tend to circulate back to the self-interest of academics and the agendas that promote our own wellbeing, rather than those of the populations we study. Much of this is inevitable if we want to obtain jobs and be successful in our careers. But this means we also need to engage in constant re-evaluation of our work and remind ourselves of our responsibilities to the wider world. Only then will we follow through on the trajectories that will ensure that we have helped to make things matter and have made a genuine contribution to countering the regressive and repressive forces that currently challenge us all.

\section{Acknowledgements}

The Why We Post project was funded by the European Research Council, ERC-2011-Advanced Grant no. 295486. The ASSA project was funded by 
the European Research Council, ERC-2016-Advanced Grant no. 740472. The authors are hugely grateful to all the other members of the two project teams, since all the work reported here comes equally from the work of those teams. We are also grateful to our research participants from all over the world whose time and support have provided the evidence for these projects.

\section{Notes}

1141 videos are available to watch on the Why We Post YouTube channel: www. youtube.com/channel/UCeaS-I5mRr7Hqf41wO5ntSQ.

2 www.ucl.ac.uk/why-we-post.

3 https://about.jstor.org/news/the-top-10-most-popular-oa-ebooks-in-jstor/.

4 https://openedu.ru/course/hse/WEPOST/.

5 Oxford Cambridge and RSA (OCR) is an awarding body that provides qualifications for people of all ages and abilities.

6 https://sociology.hku.hk/schools-resources/impact-social-media/.

7 www.ucl.ac.uk/anthropology/assa. 